\title{
Free Quantum Fields in 4D and Calabi-Yau Spaces
}

\author{
Robert de Mello Koch, ${ }^{1, *}$ Phumudzo Rabambi, ${ }^{1, \dagger}$ Randle Rabe, ${ }^{1, \$}$ and Sanjaye Ramgoolam ${ }^{1,2, \$}$ \\ ${ }^{1}$ School of Physics and Mandelstam Institute for Theoretical Physics, University of Witwatersrand, Wits, 2050, South Africa \\ ${ }^{2}$ Centre for Research in String Theory and School of Physics and Astronomy, Queen Mary University of London, \\ Mile End Road, London E1 4NS United Kingdom
}

(Received 11 May 2017; revised manuscript received 16 August 2017; published 16 October 2017)

\begin{abstract}
We develop general counting formulas for primary fields in free four dimensional (4D) scalar conformal field theory (CFT). Using a duality map between primary operators in scalar field theory and multivariable polynomial functions subject to differential constraints, we identify a sector of holomorphic primary fields corresponding to polynomial functions on a class of permutation orbifolds. These orbifolds have palindromic Hilbert series, which indicates they are Calabi-Yau orbifolds. We construct the unique top-dimensional holomorphic form expected from the Calabi-Yau property. This sector includes and extends previous constructions of infinite families of primary fields. We sketch the generalization of these results to free $4 \mathrm{D}$ vector and matrix CFTs.
\end{abstract}

DOI: 10.1103/PhysRevLett.119.161602

Introduction.-In Ref. [1] we started a program of describing the discrete combinatoric data of four dimensional conformal field theories (CFT4) using the framework of $S O(4,2)$ invariant $2 \mathrm{D}$ topological field theory (TFT2). TFT2 associates state spaces to circles and the operator product expansion of the 4D CFT determines amplitudes for 3-holed spheres. We described how the associativity conditions of 2D TFT are satisfied by the correlators of free scalar CFT4. We initiated the investigation of $S O(4,2)$ invariant TFT2 as an approach to perturbative field theory in Ref. [2], making contact with the equivariant interpretation of conformal Feynman integrals in mathematical work [3]. In this Letter we return to free scalar CFT4 and develop the concrete counting and construction of primary fields, which gives the decomposition of the state space of the 2D TFT in terms of $S O(4,2)$ representations. While two dimensional conformal field theories have infinite dimensional symmetries which allow, in the case of rational CFTs, all the local operators to fall into finitely many representations of the symmetry algebra, the complete description of local operators in even the simplest free field CFT4s is an important challenge, with implications for a variety of problems in theoretical physics. Such a complete description would amount to knowing the spectrum of any postulated holographic dual. For example, while the free $O(N)$ vector model in three dimensions has a known holographic dual [4-6], finding such a dual in four dimensions is an open problem. A complete characterization of the local operators is also likely to have implications for the physics of QCD and of the interacting scalar field theory at the WilsonFischer fixed point. Elegant arguments have been found which relate explicit information on the combinatorics of primary fields and OPE coefficients of free CFT4 to observables in the epsilon expansion [7-11]. Explicit constructions of restricted classes of primary fields in the free limit of QCD is known to have applications in the computation of anomalous dimensions of operators (see Ref. [12]). In this Letter, we present new results generalizing existing constructions of primary fields in the free scalar field theory, in $O(N)$ vector models as well as matrix models in four dimensions. These generalizations define an "extremal sector," characterized in terms of $S O(4,2)$ representation theory. The extremal sector is found to have a surprising Calabi-Yau structure, a geometrical structure arising from an intriguing interplay between $S O(4,2)$ representation theory and symmetric groups.

In radial quantization of CFTs [13], local operators at a point correspond to quantum states. The two point function yields an inner product on the quantum states and the scaling operator is the Hamiltonian. Focusing on the local operators which are primary fields constructed from $n$ copies of the elementary field $\phi$ in free scalar theory, we have an inner product and a Hamiltonian. Alongside these elements of radial quantization, we use a map between derivatives $\partial_{\mu} \rightarrow x_{\mu}$, which allows us to work with representations of $S O(4,2)$ on polynomials [see Ref. [3] for detailed treatment of $S O(4,2)$ reps from this perspective]. We thus map the problem of constructing primary fields to a problem in many body quantum mechanics of $n$ bosonic particles in $\mathbb{R}^{4}$, which has an inner product and Hamiltonian coming from radial quantization. Concretely, we have the problem of solving a system of linear constraints for functions $\Psi\left(x_{\mu}^{I}\right)$, with an associated counting problem of the dimensions of spaces of solutions as a function of the quantum mechanical energy corresponding to the degree of the polynomials. This system of equations is given in Eq. (16). One of these constraints is a harmonicity condition in $\left(\mathbb{R}^{4}\right)^{\times n}$. A large class of solutions is obtained by choosing a complex structure $(z, w)$ on $\mathbb{R}^{4}=\mathbb{C}^{2}$ and restricting to holomorphic 
solutions. The special case where the functions depend on a single complex variable makes contact with previously available explicit construction of primary fields in the literature. The reduction of the second order constraint to first order holomorphic conditions has the intriguing consequence that these primary fields have a closed ring structure. The associated generating functions have palindromicity properties due to the fact that the primary fields correspond to functions on the Calabi-Yau orbifolds

$$
\left(\mathbb{C}^{2}\right)^{n} /\left(\mathbb{C}^{2} \times S_{n}\right),
$$

which can also be written as

$$
\left(\mathbb{C}^{n} / \mathbb{C} \times \mathbb{C}^{n} / \mathbb{C}\right) / S_{n},
$$

where $n$ is the number of elementary fields $\phi$. A generalization of our discussion to the $O(N)$ vector model shows that the holomorphic singlet primary fields correspond to functions on the Calabi-Yau orbifold

$\left(\mathbb{C}^{2}\right)^{2 n} /\left(\mathbb{C}^{2} \times S_{n}\left[S_{2}\right]\right)=\left(\mathbb{C}^{2 n} / \mathbb{C} \times \mathbb{C}^{2 n} / \mathbb{C}\right) / S_{n}\left[S_{2}\right]$,

where $S_{n}\left[S_{2}\right]$ is a wreath product subgroup of $S_{2 n}$. For the matrix model in which $\phi$ transforms in the adjoint of $U(N)$, we find holomorphic primaries corresponding to polynomial functions on

$$
\left[\left(\mathbb{C}^{2}\right)^{n} \times S_{n}\right] /\left(\mathbb{C}^{2} \times S_{n}\right),
$$

which can also be written as

$$
\left(\mathbb{C}^{n} / \mathbb{C} \times \mathbb{C}^{n} / \mathbb{C} \times S_{n}\right) / S_{n} .
$$

Multivariable Polynomial (many-body) representation of $S O(4,2)$. - In radial quantization, the scalar field has a mode expansion given by

$\phi\left(x_{\mu}\right)=\sum_{l=0}^{\infty} \sum_{m \in V_{l}} a_{l, m}^{\dagger} Y_{l, m}(x)+\sum_{l=0}^{\infty} \sum_{m \in V_{l}} a_{l, m}|x|^{-2} Y_{l, m}\left(x^{\prime}\right)$.

$V_{l}$ is the representation of $S O(4)$ corresponding to symmetric traceless tensors of rank $l$. The index $m$ runs over a basis for this vector space. Acting on the vacuum state $|0\rangle$ (which is, by definition, annihilated by the $a_{l, m}$ ) with a local operator $\partial_{\mu_{1}} \ldots \partial_{\mu_{k}} \phi$ and taking the limit $x \rightarrow 0$, we get a state. Taking the dual of this state and pairing with $\phi(x)|0\rangle$ we get a polynomial. Thus, there is a map

$$
\partial_{\mu_{1}} \ldots \partial_{\mu_{k}} \phi \leftrightarrow P_{\mu_{1}} \ldots P_{\mu_{k}} \cdot 1,
$$

where [1]

$$
P_{\mu}=x^{2} \partial_{\mu}-2 x_{\mu} x . \partial-2 x_{\mu} .
$$

The scalar field itself maps to 1. The free field in Eq. (6) satisfies the equation of motion $\partial_{\mu} \partial_{\mu} \phi=0$. Correspondingly, $P_{\mu} P_{\mu}=x^{4} \partial^{2}$ annihilates 1 . When considering operators constructed using $n$ fields, we have a representation of the conformal group on polynomials in variables $x_{\mu}^{I}$, where $I$ ranges from 1 to $n$. The generators for special conformal transformations and translations are [1]

$$
\begin{gathered}
K_{\mu}=\sum_{I=1}^{n} \frac{\partial}{\partial x_{\mu}^{I}}, \\
P_{\mu}=\sum_{I=1}^{n}\left(x^{I \rho} x_{\rho}^{I} \frac{\partial}{x_{\mu}^{I}}-2 x_{\mu}^{I} x_{\rho}^{I} \frac{\partial}{x_{\rho}^{I}}-2 x_{\mu}^{I}\right) .
\end{gathered}
$$

The remaining generators are determined by the $\operatorname{so}(4,2)$ algebra. The $x_{\mu}^{I}$ can be considered as the coordinates of $n$ particles. The construction of primaries using $n$ copies of the elementary field $\phi$ is therefore mapped to a manybody quantum mechanics problem with $n$ particles. Tracelessness can be implemented [14,15] using variables $z x^{I}=z^{\mu} x_{\mu}^{I}$ with null $z^{\mu}: z^{\mu} z_{\mu}=0$. Any polynomial in $z x^{I}$ gives a traceless symmetric polynomial in $x_{\mu}^{I}$ after the $z^{\mu} \mathrm{S}$ are stripped away. The translation between polynomials and operators is

$$
(z \partial)^{k} \phi \leftrightarrow(-1)^{k} 2^{k} k !(z x)^{k} .
$$

This construction is not general: there are primaries that are not symmetric in their indices and so cannot be represented as a polynomial in $z x$. For the general discussion, introduce projectors from symmetric tensors to traceless symmetric tensors. For example, for tensors of rank 2 and 3 we have

$$
\begin{aligned}
S_{\mu \nu}^{\alpha \beta} & =\delta_{\mu}^{\alpha} \delta_{\nu}^{\beta}-\frac{1}{4} \delta_{\mu \nu} \delta^{\alpha \beta}, \\
S_{\mu \nu \rho}^{\alpha \beta \gamma} & =\delta_{\mu}^{\alpha} \delta_{\nu}^{\beta} \delta_{\rho}^{\gamma}-\frac{1}{6}\left(\delta_{\mu \nu} \delta^{\alpha \beta} \delta_{\rho}^{\gamma}+\delta_{\mu \rho} \delta^{\alpha \gamma} \delta_{\nu}^{\beta}+\delta_{\mu}^{\alpha} \delta^{\beta \gamma} \delta_{\nu \rho}\right) .
\end{aligned}
$$

We recognize that these are projectors in the Brauer algebra of tensor space operators which commute with $S O(4)$ [16]

$$
\begin{aligned}
& S^{(2)}=1-\frac{C_{12}}{4}, \\
& S^{(3)}=1-\frac{1}{6}\left(C_{12}+C_{13}+C_{23}\right) .
\end{aligned}
$$

The $C_{i j}$ are linear operators with matrix elements as in Eq. (12). They satisfy

$$
\left(S^{(n)}\right)^{2} P_{n}=S^{(n)} P_{n},
$$

where

$$
P_{n}=\frac{1}{n !} \sum_{\sigma \in S_{n}} \sigma .
$$

The projector property along with the property that they start with 1 completely determines these elements of the Brauer algebra. In general,

$$
P_{\mu_{1}} \ldots P_{\mu_{n}} \times 1=(-1)^{n} 2^{n} n !\left(S^{(n)}\right)_{\mu_{1} \ldots \mu_{n}}^{\nu_{1} \ldots \nu_{n}} x_{\nu_{1}} \ldots x_{\nu_{n}} .
$$

The multiplication (14) is in the Brauer algebra, where loops are assigned the value of 4 . The factor on the righthand side above is obtained by deriving an obvious 
recursion formula. Note that the term $x^{2} \partial_{\mu}$ in $P_{\mu}$ does not raise the rank of the tensor. The other two terms contribute to the recursion.

States at dimension $n+k$ in $V^{\otimes n}$ correspond to polynomials in $x_{\mu}^{I}$ of degree $k$. Primaries at dimension $n+k$ are degree $k$ polynomials $\Psi\left(x_{\mu}^{I}\right)$ with the conditions

$$
\begin{aligned}
K_{\mu} \Psi\left(x_{\mu}^{I}\right) & =\sum_{I} \frac{\partial}{\partial x_{\mu}^{I}} \Psi\left(x_{\mu}^{I}\right)=0, \\
\mathcal{L}_{I} \Psi\left(x_{\mu}^{I}\right) & =\sum_{\mu} \frac{\partial}{\partial x_{\mu}^{I}} \frac{\partial}{\partial x_{\mu}^{I}} \Psi\left(x_{\mu}^{I}\right)=0, \\
\Psi\left(x_{\mu}^{I}\right) & =\Psi\left(x_{\mu}^{\sigma(I)}\right) .
\end{aligned}
$$

The first condition says the special conformal generators annihilate a primary operator. The second condition implements the free scalar equation of motion. The last condition imposes $S_{n}$ invariance, to implement bosonic statistics of the scalar field.

We find it useful to employ the complex coordinates

$$
\begin{array}{ll}
z=x_{1}+i x_{2}, & w=x_{3}+i x_{4}, \\
\bar{z}=x_{1}-i x_{2}, & \bar{w}=x_{3}-i x_{4},
\end{array}
$$

which have the following $\left(j_{L}^{3}, j_{R}^{3}\right)$ charge assignments

$$
\begin{aligned}
& z \leftrightarrow\left(\frac{1}{2}, \frac{1}{2}\right), \quad \bar{z} \leftrightarrow\left(-\frac{1}{2},-\frac{1}{2}\right), \\
& w \leftrightarrow\left(\frac{1}{2},-\frac{1}{2}\right), \quad \bar{w} \leftrightarrow\left(-\frac{1}{2}, \frac{1}{2}\right) .
\end{aligned}
$$

This amounts to choosing an isomorphism between $\mathbb{R}^{4}$ and $\mathbb{C} \times \mathbb{C}$. We will construct a class of primaries corresponding to holomorphic polynomial functions on

$$
\mathbb{C}^{2 n} /\left(\mathbb{C}^{2} \times S_{n}\right)
$$

Counting with $S O(4,2)$ characters.-The number $N_{\left[\Delta, j_{1}, j_{2}\right]}$ of primary operators, of dimension $\Delta$ and spin $\left(j_{1}, j_{2}\right)$ built out of $n$ scalar fields $\phi$ is obtained by expanding the generating function

$$
G_{n}(s, x, y)=\sum_{m, j_{1}, j_{2}} N_{\left[m, j_{1}, j_{2}\right]} s^{m} x^{j_{1}} y^{j_{2}} .
$$

The generating function is given by (take $n \geq 3$ to avoid complications associated to null states)

$$
\begin{aligned}
G_{n}(s, x, y)= & {\left[\left(1-\frac{1}{x}\right)\left(1-\frac{1}{y}\right) Z_{n}(s, x, y)(1-s \sqrt{x y})\right.} \\
& \left.\left(1-s \sqrt{\frac{x}{y}}\right)\left(1-s \sqrt{\frac{y}{x}}\right)\left(1-\frac{s}{\sqrt{x y}}\right)\right]_{\geq},
\end{aligned}
$$

where $Z_{n}(s, x, y)$ is defined by

$$
\prod_{q=0}^{\infty} \prod_{a=-\frac{q}{2}}^{\frac{q}{2}} \prod_{b=-\frac{q}{2}}^{\frac{q}{2}} \frac{1}{1-t s^{q+1} x^{a} y^{b}}=\sum_{n=0}^{\infty} t^{n} Z_{n}(s, x, y) .
$$

This is obtained by constructing the character for the symmetric product of $n$ copies of the representation of the scalar field, and decomposing into $S O(4,2)$ irreps $[17,18]$.

We can specialize this counting formula. Consider the leading twist fields, with $\left[\Delta, j_{1}, j_{2}\right]=\{n+q,[(q / 2),(q / 2)]\}$. This is a complete spin multiplet. The highest spin primary corresponds to a polynomial in $z$. For counting these primaries, the general formulas given above reduce to

$$
G_{n}^{z}(s, x, y)=\left[Z_{n}^{z}(s, x, y)(1-s \sqrt{x y})\right],
$$

where

$$
\prod_{q=0}^{\infty} \frac{1}{1-t s^{q+1} x^{\frac{q}{2}} y^{\frac{q}{2}}}=\sum_{n=0}^{\infty} t^{n} Z_{n}^{z}(s, x, y) .
$$

Using the simplified formulas we have

$$
G_{n}^{\max }(s)=\frac{s^{n}}{\left(1-s^{2}\right)\left(1-s^{3}\right) \ldots\left(1-s^{n}\right)} .
$$

Note the close connection to multiplicities of $V_{\Lambda=n+k}^{S L(2)} \otimes V_{[n]}^{S_{n}}$, which is the coefficient of $q^{k}$ in

$$
\prod_{i=2}^{n} \frac{1}{1-q^{i}}
$$

The result (25) was also recently obtained in Ref. [19].

A more general counting involves polynomials of $z_{\mu}^{I}$ and $w_{\mu}^{I}$. We denote these as extremal primaries, since they have $\left(s, j_{L}, j_{R}\right)=\left[n+q,(q / 2), j_{R}\right]$. In this case

$$
\begin{aligned}
G_{n}^{z, w}(s, x, y)= & {\left[\left(1-\frac{1}{y}\right)(1-s \sqrt{x y})\right.} \\
& \left.(1-s \sqrt{x / y}) Z_{n}(s, x, y)\right]_{\geq},
\end{aligned}
$$

where

$$
\prod_{q=0}^{\infty} \prod_{m=0}^{q} \frac{1}{\left(1-t s^{q+1} x^{q / 2} y^{m-q / 2}\right)}=\sum_{n=0}^{\infty} t^{n} Z_{n}^{z, w}(s, x, y) .
$$

As explained in more detail in Sec. IV,

$Z_{n}^{z, w}(s, x, y)=s^{n} \sum_{\Lambda_{1}} Z_{\mathrm{SH}}\left(s \sqrt{x y}, \Lambda_{1}\right) Z_{\mathrm{SH}}\left(s \sqrt{\frac{x}{y}}, \Lambda_{1}\right)$,

where $\Lambda_{1}$ is a partition of $n$, and $Z_{\mathrm{SH}}(q)$ is given in Eq. (33). Using these formulas, one finds, for $n=3$

$$
\begin{aligned}
Z_{3}^{z, w} & =\frac{s^{3}\left[s^{6} x^{3}+s^{4} x^{2}+s^{2} x+1+s^{3} x^{\frac{3}{2}}\left(\sqrt{y}+\frac{1}{\sqrt{y}}\right)\right]}{\left(1-s^{2} x y\right)\left(1-s^{3}(x y)^{\frac{3}{2}}\right)\left(1-s^{2} \frac{x}{y}\right)\left(1-\frac{s^{3} x^{\frac{3}{2}}}{y^{\frac{3}{2}}}\right)} . \\
G_{3}^{z, w} & =\frac{s^{3}\left(1+s^{5} x^{\frac{5}{2}} y^{\frac{3}{2}}\right)}{\left(1-s^{4} x^{2}\right)\left(1-s^{3} \sqrt{x^{3} y^{3}}\right)\left(1-s^{2} x y\right)} .
\end{aligned}
$$


Computing $Z_{4}(s, x, y)$ in the same way we find

$$
G_{4}^{z, w}=\frac{s^{4} R(s, x, y)}{D(s, x, y)}
$$

where

$$
\begin{aligned}
R(s, x, y)= & 1+s^{5} x^{\frac{5}{2}}\left[\sqrt{y}+s^{3} x^{\frac{3}{2}} y+s^{5} x^{\frac{5}{2}} y+y^{3}-s^{6} x^{3} y^{\frac{5}{2}}\right. \\
& -s^{8} x^{4} y^{\frac{5}{2}}-s^{16} x^{8} y^{\frac{7}{2}}-s^{11} x^{\frac{11}{2}} y^{2}(1+y) \\
& +s^{7} x^{\frac{7}{2}}\left(1-y^{2}\right)+s^{4} x^{2} y^{\frac{3}{2}}\left(1-y^{2}\right) \\
& +s^{2} x \sqrt{y}\left(1+y^{2}\right)-s^{9} x^{\frac{9}{2}} y\left(1+y^{2}\right) \\
& \left.-s^{10} x^{5} y^{\frac{3}{2}}\left(1+y-y^{2}\right)-s \sqrt{x}\left(1-y-y^{2}\right)\right] . \\
D(s, x, y)= & \left(1-s^{2} x y\right)\left(1-s^{3} x^{\frac{3}{2}} y^{\frac{3}{2}}\right)\left(1-s^{4} x^{2} y^{2}\right) \\
& \left(1-s^{4} x^{2}\right)\left(1-s^{6} x^{3}\right)\left(1-s^{8} x^{4}\right) .
\end{aligned}
$$

Similar constructions with the pairs $(z, \bar{w}),(\bar{z}, w),(\bar{z}, \bar{w})$ are possible.

Counting and construction with symmetric groups.The counting formulas derived in Sec. III can be used to construct families of primary operators. The coordinates $x_{\mu}^{I}$, $I=1, \ldots, n$ admit a natural action of $S_{n}$. To satisfy the first of Eq. (16), build $n-1$ translation invariant "relative coordinates" given by the successive differences $x_{\mu}^{(a)}=$ $x_{\mu}^{a}-x_{\mu}^{a+1}$. Using the complex coordinates $z, w$ on $\mathbb{R}^{4}=\mathbb{C}^{2}$, we have $\left(z_{I}, w_{I}\right)$ on $\left(\mathbb{R}^{4}\right)^{n}=\left(\mathbb{C}^{2}\right)^{n}$. These differences span the $S_{n}$ irrep labeled by hook Young diagram with row lengths $[n-1,1]$. A more convenient basis which connects with Young's orthonormal representation is useful for computations (see Ref. [20] for this basis). Using complex variables we have $z^{(a)}, \bar{z}^{(a)}, w^{(a)}$, and $\bar{w}^{(a)}$, which each transform in the irrep $[n-1,1] \equiv V_{H}$. Products $z^{\left(a_{1}\right)} z^{\left(a_{2}\right)} \cdots z^{\left(a_{k}\right)}$ are in the $V_{H}^{\otimes k}$ tensor product representation of $S_{n}$. Any polynomial in the hook variables automatically obeys the first two constraints of Eq. (16). This follows since the Laplacian in the second equation is

$$
\left(\frac{\partial^{2}}{\partial z^{I} \partial \bar{z}^{I}}+\frac{\partial^{2}}{\partial w^{I} \partial \bar{w}^{I}}\right) \Psi=0
$$

The only thing left is to project to the $S_{n}$ invariant subspace of $V_{H}^{\otimes k}$. The matrices representing the $k$-fold tensor product are

$$
\left[\Gamma_{k}^{n}(\sigma)\right]_{a_{1}, \ldots, a_{k} ; b_{1}, \ldots, b_{k}}=\Gamma_{a_{1}, b_{1}}(\sigma) \ldots \Gamma_{a_{k}, b_{k}}(\sigma),
$$

where $\Gamma_{a, b}(\sigma)$ are matrices representing $S_{n}$ in an orthogonal basis of $[n-1,1]$. We can project to the invariants by averaging over the group

$$
P_{a_{1} a_{2} \cdots a_{k}}=\frac{1}{n !} \sum_{\sigma \in S_{n}}\left[\Gamma_{k}(\sigma)\right]_{a_{1} a_{2} \cdots a_{k}, b_{1} b_{2} \cdots b_{k}} Z^{\left(b_{1}\right)} \cdots Z^{\left(b_{k}\right)} .
$$

The above expression gives $\sum_{i} \hat{n}_{i} P_{i}(z)$, where $\hat{n}_{i}$ are unit vectors and $P_{i}\left(z_{1}, \ldots, z_{n}\right)$ are the polynomials we want.
By considering all possible degrees $k \in\{0,1,2, \ldots\}$ we have a ring. These primaries have a ring structure, since they obey a stronger linear version of the Laplacian condition, which means that a product of solutions is also a solution to the constraints. The counting formula (25) gives the Hilbert series for holomorphic functions on $\left(\mathbb{C}^{n} / \mathbb{C}\right) / S_{n}$. The quotient by $\mathbb{C}$ is effected by the first of Eq. (16) which sets the center of mass momentum of the many body wave function to zero. The orbifold by $S_{n}$ is the symmetry condition in Eq. (16). Using properties of Hilbert series, it follows that the ring at hand has $n-1$ generators, which can be described explicitly [18].

The construction is easily extended to polynomials of holomorphic coordinates $z^{I}$ and $w^{I}$. Use hook variables $Z^{(a)}$, $W^{(a)}$. The products $Z^{\left(a_{1}\right)} \ldots Z^{\left(a_{k}\right)} W^{\left(a_{k_{1}}\right)} \ldots W^{\left(a_{k+l}\right)}$ belong to a subspace of the representation $V_{H}^{\otimes k} \otimes V_{H}^{\otimes l}$ of $S_{n}$, which we will characterize in terms of representation theory. Consider the expansions in terms of $S_{n} \times S_{k}$ irreps

$$
\begin{aligned}
& V_{H}^{\otimes k}=\bigoplus_{\Lambda_{1} \vdash n, \Lambda_{2} \vdash k} V_{\Lambda_{1}}^{\left(S_{n}\right)} \otimes V_{\Lambda_{2}}^{\left(S_{k}\right)} \otimes V_{\Lambda_{1}, \Lambda_{2}}^{\operatorname{Com}\left(S_{n} \times S_{k}\right),} \\
& V_{H}^{\otimes l}=\bigoplus_{\Lambda_{3} \vdash n, \Lambda_{4} \vdash l} V_{\Lambda_{3}}^{\left(S_{n}\right)} \otimes V_{\Lambda_{4}}^{\left(S_{l}\right)} \otimes V_{\Lambda_{3}, \Lambda_{4}}^{\operatorname{Com}\left(S_{n} \times S_{l}\right) .}
\end{aligned}
$$

Multiplicities are given by dimensions of irreps of the commutants $\operatorname{Com}\left(S_{n} \times S_{k}\right)$ in $V_{H}^{\otimes k}$. Since the $Z$ and $W$ variables are commuting, the monomials belong to the trivial irreps $\Lambda_{2}=[k] \otimes \Lambda_{4}=[l]$ of $S_{k} \times S_{l}$. To satisfy the third constraint, project to $S_{n}$ invariants in $V_{H}^{\otimes k} \otimes V_{H}^{\otimes l}$. This constrains $\Lambda_{3}=\Lambda_{1}$. So the number of $S_{k} \times S_{l} \times S_{n}$ invariants is

$$
\sum_{\Lambda_{1} \vdash n} \operatorname{Mult}\left(\Lambda_{1},[k] ; S_{n} \times S_{k}\right) \operatorname{Mult}\left(\Lambda_{1},[l] ; S_{n} \times S_{l}\right) .
$$

The expansions (32) are explained further and used in the construction of orthogonal bases of local operators in Ref. [20]. The generating functions for these multiplicities are derived in Ref. [20]. $\operatorname{Mult}\left(\Lambda_{1},[k] ; S_{n} \times S_{k}\right) \equiv Z_{\mathrm{SH}}^{k}$ is the coefficient of $q^{k}$ in

$$
\begin{aligned}
Z_{\mathrm{SH}}\left(q ; \Lambda_{1}\right) & =(1-q) q^{\left\{\left[\sum_{i} c_{i}\left(c_{i}-1\right)\right] / 2\right\}} \prod_{b} \frac{1}{\left(1-q^{h_{b}}\right)} \\
& =\sum_{k} q^{k} Z_{\mathrm{SH}}^{k}\left(\Lambda_{1}\right) .
\end{aligned}
$$

Here, $c_{i}$ is the length of the $i$ th column in $\Lambda_{1}, b$ runs over boxes in the Young diagram $\Lambda_{1}$, and $h_{b}$ is the hook length of the box $b$. Thus, for the number of primaries constructed from $z_{i}, w_{i}$ we get

$$
\sum_{\Lambda_{1} \vdash n} Z_{\mathrm{SH}}^{k}\left(\Lambda_{1}\right) Z_{\mathrm{SH}}^{l}\left(\Lambda_{1}\right)
$$

These are primaries of weight $n+k+l$, with $\left(J_{3}^{L}, J_{3}^{R}\right)=$ $\{[(k+l) / 2],[(k-l) / 2]\}$. We can also show directly that $Z_{n}(s, x, y)$ in Eq. (27) is a sum over irreps $\Lambda_{1}$ of $S_{n}$ as above. 
Thus,

$$
\begin{aligned}
Z_{n}(s, x, y) & =\sum_{\Lambda_{1} \vdash n} \sum_{k, l} Z_{\mathrm{SH}}^{k}\left(\Lambda_{1}\right) Z_{\mathrm{SH}}^{l}\left(\Lambda_{1}\right) s^{n+k+l} x^{[(k+l) / 2]} y^{[(k-l) / 2]} \\
& =s^{n} \sum_{\Lambda_{1} \vdash n} Z_{\mathrm{SH}}\left(q=s \sqrt{x y}, \Lambda_{1}\right) Z_{\mathrm{SH}}\left(q=s \sqrt{x / y}, \Lambda_{1}\right) .
\end{aligned}
$$

Using the generating function (33), we get the rational expressions for $Z_{3}(s, x, y), Z_{4}(s, x, y)$ used in Sec. III, by explicitly doing the sum over $\Lambda_{1}$.

This structure in the counting problem provides an explicit construction formula. First, decompose the $z$ and $w$ polynomials into definite $S_{n}$ irreps. The projector onto irrep $r$ from the tensor product of $k$ copies of the hook is

$$
P_{a_{1} \ldots a_{k}, b_{1} \ldots b_{k}}^{\Lambda_{1}}=\frac{1}{n !} \sum_{\sigma \in S_{n}} \chi_{\Lambda_{1}}(\sigma)\left[\Gamma_{k}^{n}(\sigma)\right]_{a_{1} \ldots a_{k}, b_{1} \ldots b_{k} .}
$$

We also need the projection onto the symmetric irrep

$$
P_{a_{1} \ldots a_{n}, b_{1} \ldots b_{n}}=\frac{1}{n !} \sum_{\sigma \in S_{n}}\left[\Gamma_{k}^{n}(\sigma)\right]_{a_{1} \ldots a_{n}, b_{1} \ldots b_{n} .}
$$

Using these two projectors, the polynomials constructed using two holomorphic variables are

$$
\begin{aligned}
\sum_{A} P_{A}(\vec{z}, \vec{w}) \hat{n}_{e_{1} \ldots e_{k+l}}^{A}= & P_{e_{1} \ldots e_{k+l}, a_{1} \ldots a_{k} c_{1} \ldots c_{l}} \\
& \times P_{a_{1} \ldots a_{k}, b_{1} \ldots b_{k}}^{r} P_{c_{1} \ldots c_{l}, d_{1} \ldots d_{l}}^{r} \\
& \times Z^{\left(b_{1}\right)} \ldots Z^{\left(b_{k}\right)} W^{\left(d_{1}\right)} \ldots W^{\left(d_{l}\right)},
\end{aligned}
$$

where $\hat{n}^{A}$ are unit vectors and $P_{A}\left(z^{I}, w^{I}\right)$ are the polynomials corresponding to primary fields. These polynomials satisfy all the conditions in Eq. (16). They satisfy stronger linear equations

$$
\partial_{\bar{w}^{J}} P_{A}\left(z^{I}, w^{I}\right)=0 ; \quad \partial_{\bar{z}^{J}} P_{A}\left(z^{I}, w^{I}\right)=0,
$$

which imply the Laplacian conditions. As a result, taking all possible $k, l$, we have a space of solutions to the constraints which forms a ring due to the Leibniz rule for products of functions. This is the polynomial ring of holomorphic functions for

$$
\left[\left(\mathbb{C}^{n} / \mathbb{C}\right) \times\left(\mathbb{C}^{n} / \mathbb{C}\right)\right] / S_{n}
$$

Using generalities about Hilbert series for algebraic varieties (see Refs. [21,22] for applications in the context of moduli spaces of SUSY gauge theories), we see from Eq. (29) that for $n=3$ the polynomials $P_{A}(z, w)$ are a finitely generated polynomial ring with 3 generators. The explicit constructions described above allow us to identify the generators $\left(z_{i j} \equiv z_{i}-z_{j}\right)$

$$
\begin{aligned}
\left(z_{12}\right)^{2 k}+\left(z_{13}\right)^{2 k}+\left(z_{23}\right)^{2 k} & \leftrightarrow\left(s^{2} x y\right)^{k}, \\
\left(z_{13}+z_{23}\right)^{k}\left(z_{31}+z_{21}\right)^{k}\left(z_{12}+z_{32}\right)^{k} & \leftrightarrow\left(s^{3} \sqrt{x^{3} y^{3}}\right)^{k}, \\
\left|\begin{array}{ccc}
w_{1} & w_{2} & w_{3} \\
z_{1} & z_{2} & z_{3} \\
1 & 1 & 1
\end{array}\right|^{2 k} & \leftrightarrow\left(s^{4} x^{2}\right)^{k} .
\end{aligned}
$$

This is explained in more detail in the forthcoming paper [18].

The Hilbert series associated to the counting of primary fields ensures a palindromic property of the numerators. This can be verified for $Z_{3}(s, x, y), Z_{4}(s, x, y)$. A general property of the numerators

$$
Q_{n}(s, x, y)=\sum_{k=0}^{D} a_{k}(x, y) s^{k}
$$

is that $a_{D-k}(x, y)=a_{k}(x, y)$. A direct proof using the combinatoric expressions like Eq. (28) in terms of symmetric group representation theory data, is given in Ref. [18]. The theorem of Stanley [23] suggests that these orbifolds are Calabi-Yau. This can be explicitly demonstrated by constructing the top form and verifying that it is nowhere vanishing [18].

The above argument starting from counting to motivate a construction of the primary operators and then an associated Calabi-Yau geometry goes through when the single scalar is generalized to the $O(N)$ vector model and to the free $U(N)$ gauge theory with $\phi$ a matrix in the adjoint. The relevant geometries are the Calabi-Yau orbifolds

$$
\left(\mathbb{C}^{2 n} / \mathbb{C} \times \mathbb{C}^{2 n} / \mathbb{C}\right) / S_{n}\left[S_{2}\right]
$$

and

$$
\left(\mathbb{C}^{n} / \mathbb{C} \times \mathbb{C}^{n} / \mathbb{C} \times S_{n}\right) / S_{n},
$$

respectively. It is fascinating that nontrivial properties of the combinatorics of primary fields in free four dimensional conformal field theory is related to the geometry of CalabiYau orbifolds (38), (41), and (42). The interplay between representation theory of symmetric groups and of the conformal group $S O(4,2)$ which has led to this result is likely to have generalizations in other dimensions and in other physics problems involving the representation theory of conformal groups.

This work of R. d M. K., P. R., and R. R. is supported by the South African Research Chairs Initiative of the Department of Science and Technology and National Research Foundation as well as funds recieved from the National Institute for Theoretical Physics (NITheP). S. R. is supported by the STFC consolidated Grant No. ST/ L000415/1 String Theory, Gauge Theory \& Duality, and a Visiting Professorship at the University of the Witwatersrand, funded by a Simons Foundation grant held at the Mandelstam Institute for Theoretical Physics. 
*robert@neo.phys.wits.ac.za

†57990@students.wits.ac.za

†randlerabe@gmail.com

\$s.ramgoolam@qmul.ac.uk

[1] R. de Mello Koch and S. Ramgoolam, Nucl. Phys. B890, 302 (2014).

[2] R. de Mello Koch and S. Ramgoolam, J. High Energy Phys. 03 (2016) 165.

[3] I. Frenkel and M. Libine, Adv. Math. 218, 1806 (2008).

[4] M. A. Vasiliev, Int. J. Mod. Phys. D 05, 763 (1996).

[5] I. R. Klebanov and A. M. Polyakov, Phys. Lett. B 550, 213 (2002).

[6] E. Sezgin and P. Sundell, J. High Energy Phys. 07 (2005) 044.

[7] S. Rychkov and Z. M. Tan, J. Phys. A 48, 29FT01 (2015).

[8] P. Basu and C. Krishnan, J. High Energy Phys. 11 (2015) 040.

[9] S. Ghosh, R. K. Gupta, K. Jaswin, and A. A. Nizami, J. High Energy Phys. 03 (2016) 174.

[10] A. Raju, J. High Energy Phys. 10 (2016) 097.

[11] K. Nii, J. High Energy Phys. 07 (2016) 107.

[12] G. P. Korchemsky, Lett. Math. Phys. 99, 425 (2012).
[13] S. Rychkov, EPFL Lectures on Conformal Field Theory in $D \geq 3$ Dimensions, Springer Briefs in Physics (Springer, New York, 2017).

[14] V. K. Dobrev, V. B. Petkova, S. G. Petrova, and I. T. Todorov, Phys. Rev. D 13, 887 (1976).

[15] M. S. Costa, J. Penedones, D. Poland, and S. Rychkov, J. High Energy Phys. 11 (2011) 071.

[16] R. Goodman and N. R. Wallach, Representations and Invariants of the Classical Groups (Cambridge University Press, Cambridge, England, 1998).

[17] T. H. Newton and M. Spradlin, Phys. Lett. B 672, 382 (2009).

[18] R. de Mello Koch, P. Rabambi, R. Rabe, and S. Ramgoolam, J. High Energy Phys. 08 (2017) 077.

[19] K. Roumpedakis, J. High Energy Phys. 07 (2017) 109.

[20] T. W. Brown, P. J. Heslop, and S. Ramgoolam, J. High Energy Phys. 04 (2009) 089.

[21] J. Gray, A. Hanany, Y. H. He, V. Jejjala, and N. Mekareeya, J. High Energy Phys. 05 (2008) 099.

[22] A. Hanany, N. Mekareeya, and G. Torri, Nucl. Phys. B825, 52 (2010).

[23] R. Stanley, Adv. Math. 28, 57 (1978). 